\title{
Erratum to: Variants in TNIP1, a regulator of the NF-kB pathway, found in two patients with neural tube defects
}

\author{
Francesca La Carpia $^{1}$ - Claudia Rendeli ${ }^{2}$ Clelia Molinario ${ }^{3}$ - Annamaria Milillo ${ }^{3}$. \\ Chiara Farroni $^{3}$ - Natalia Cannelli ${ }^{3}$ - Emanuele Ausili ${ }^{2}$. Valentina Paolucci ${ }^{2}$. \\ Giovanni Neri $^{3} \cdot$ Costantino Romagnoli $^{2} \cdot$ Eugenio Sangiorgi $^{3} \cdot$ Fiorella Gurrieri $^{3}$
}

Published online: 18 May 2016

(C) Springer-Verlag Berlin Heidelberg 2016

Erratum to: Childs Nervous System

DOI 10.1007/s00381-016-3087-1

In the original publication of this article, the capturing of authornames were incorrect. Authors' firstname were incorrectly presented as surname and vice-versa, except for Clelia Molinario. Here's the correct capturing of names:

Francesca La Carpia

Claudia Rendeli

Clelia Molinario

Annamaria Milillo

Chiara Farroni

Natalia Cannelli

Emanuele Ausili

Valentina Paolucci

Giovanni Neri

Costantino Romagnoli

Eugenio Sangiorgi

Fiorella Gurrieri

The online version of the original article can be found at http://dx.doi.org/ 10.1007/s00381-016-3087-1.

Fiorella Gurrieri

fiorella.gurrieri@unicatt.it

1 Department of Pathology and Cell Biology, Columbia University Medical Center, Columbia, NY, USA

2 Istituto di Pediatria, Università Cattolica del Sacro Cuore “A.

Gemelli", Rome, Italy

3 Istituto di Medicina Genomica, Università Cattolica del Sacro Cuore “A. Gemelli”, L.go Francesco Vito 1, 00168 Rome, Italy 